Running head: Mechanisms of Parental Stress

This is a preprint awaiting peer review

\title{
Mechanisms of Parental Stress During and After the COVID-19 Lockdown:
}

A two-wave longitudinal study

Miriam S. Johnson, ${ }^{1}$ Nora Skjerdingstad, ${ }^{2}$ Omid V. Ebrahimi, ${ }^{2,3}$

Asle Hoffart, ${ }^{2,3}$ Sverre Urnes Johnson ${ }^{2,3}$

${ }^{1}$ Oslo Metropolitan University, Oslo, Norway

${ }^{2}$ Department of Psychology, University of Oslo, Oslo, Norway

${ }^{3}$ Modum Bad Psychiatric Hospital, Vikersund, Norway

\section{Correspondence to:}

Miriam S. Johnson

Address: Oslo Metropolitan University, Postboks 4, St. Olavs plass. 0130 Oslo, Norway.

E-mail: mirsin@oslomet.no

\section{Citation:}

Johnson, M. S., Skjerdingstad, N., Ebrahimi, O. V., Hoffart, A., \& Johnson, S. U. (2020, December 30).

Mechanisms of Parental Stress During and After the COVID-19 Lockdown: A two-wave longitudinal study. doi:

https://doi.org/10.31234/osf.io/76pgw

Retrieved from https://psyarxiv.com/76pgw. 


\begin{abstract}
Background:

In the unpredictable times of the ongoing global coronavirus disease (COVID-19), parents worldwide are affected by stressors and strains that follow in the wake of the governmentinitiated distancing protocols.
\end{abstract}

Objective: In a two-wave longitudinal survey, we examined levels of parental perceived stress and symptoms of anxiety and depression among a sample of parents at two time points; in the midst of the strictest government-initiated physical distancing protocols following the onset of the COVID-19 pandemic (T1, $N=2868)$ and three months after the protocols discontinued (T2, $n=1489)$. Further, we investigated the levels of parental stress, anxiety, and depression relative to perceived relationship quality and anger aimed at child(ren) at the two time points, including subgroups based on age, sex, cultural background, civil status, education level, number of children in household, employment status, and pre-existing psychiatric diagnosis.

\title{
Methods and findings:
}

Parents were asked to fill out a set of validated questionnaires on the two measurement occasions. As expected, the findings indicate that the high levels of parental stress significantly decreased from T1 to T2, indicating that the cumulative stressors that parent's experiences during distancing protocols declined as a function of the phaseout of the protocols. The decrease of perceived parental stress at the two time points, was accompanied 
Running head: Mechanisms of Parental Stress

by a significant decrease in symptoms of both depression and anxiety among the participating parents. Symptoms meeting the clinical cut-off for depression (23.0\%) and generalized anxiety disorder $(23.3 \%)$ were reported among participating parents at $\mathrm{T} 1$, compared to $16.8 \%$ and $13.8 \%$ respectively at $\mathrm{T} 2$. Reduction in depression and angry at child(ren) from $\mathrm{T} 1$ to $\mathrm{T} 2$ were further associated with a reduction in perceived parental stress. In addition, relationship quality and angry at child(ren) at $\mathrm{T} 1$ predicted change in parental stress.

Conclusions: The findings underline some of the negative psychological impact of physical distancing protocols on parent's health and well-being. Parents who are facing physical distancing and remaining at home with their children may be particularly vulnerable to parental stress, anxiety and depression. Uncovering the nature of how these constructs are associated to parents and families facing social crisis, such as the ongoing pandemic, can contribute to design relevant interventions to reduce parental stress and strengthen parental coping and resilience.

Keywords: COVID-19 lockdown, parental stress, anxiety, depression, social distancing. 


\section{Introduction}

With the rapid evolvement of the global coronavirus disease (COVID-19), governments worldwide decided to close down schools and workplaces, leaving families quarantined for months to slow the spread of the virus. In these unpredictable times of a global pandemic, parents and their children are affected by a new range of stressors and strains that challenge the everyday family life and functionality. Although some parents, as with the population in general, will mobilize coping and resilience in the face of the COVID-19 threat $(1,2)$, many others will likely exacerbate existing mental health problems and contribute to the onset of new stress-related disorders (3). Initial evidence, which already indicates how the ongoing pandemic has affected the health and well-being of parents and children (4-10), probably barely scratches the surface of the potential long-term effects of the pandemic on families' health and well-being.

The unprecedented challenges of experiencing disruption of daily routines and competing demands for individuals and families, such as working from home while also educating and caring for their children, unemployment, economic hardship, and worrying about the health of their family and relatives, all in an environment of social isolation, may all contribute to fuel exacerbated stress among parents. Particular concerns have been raised about neglected and vulnerable children and children in at-risk families, who are disproportionately affected since childcare professionals and support services were suspended during the lockdown $(11,12)$. 
These concerns related to the home environment in social crisis are grounded in the robust literature on how parent-perceived stress is associated with parenting behaviors that may place undue strain on the parent-child relationship (13). In addition, stress is experienced both at the individual and at the dyadic levels (14) and both parent-child dynamic and relationship among couples are affected by home confinement. Increasingly stressed parents find it increasingly difficult to understand their child's needs and to respond sensitively (14). Moreover, parents with elevated stress accompanied by symptoms of anxiety and depressive were found less responsive to their children's needs, which in turn is a strong predictor of child abuse potential (15). Parents experiencing increased parental stress, were also found to exhibit more harsh parenting behaviors (16), which in turn increases the risk of child maltreatment. Existing research also indicates that traumatic or life-threatening events, such as natural disasters, may lead to deterioration of relationship quality due to stress and conflict (17-19) and increased experiences of domestic violence (20).

Studies of earlier social crises, which addressed levels of stress among quarantined parents, reported a high presence of psychological distress, including stress, depression, anxiety, and symptoms of post-traumatic stress $(21,22)$.

Recent cross-sectional studies provide insight into the impact of the ongoing pandemic on family dynamics and functionality by investigating how parents' and children's wellbeing, as well as mechanisms of parenting, are directly affected. One recent study among 183 parents, showed that greater COVID-19-related stressors and high anxiety and depressive symptoms were associated with higher parent-perceived stress (4). Greater parental support and perceived control during the pandemic were further associated with lower perceived stress and child abuse potential (4). A similar recent study by Spinelli and colleagues (23), which explored the effect of risk factors associated with the COVID-19 experiences on parents' and children's well-being among 854 parents, found that parents who reported more 
Running head: Mechanisms of Parental Stress

difficulties in dealing with quarantine showed more stress. Quarantine's impact on children's behavioral and emotional problems was found to be mediated by parent's individual and dyadic stress, with a stronger effect from the latter (23).

How couples, that also fulfil the roles as parents, have been affected by COVID-19 lockdown and how they cope with pandemic stress, have also been examined in some preliminary studies. A recent study among 742 American adults, found that $34 \%$ of individuals in relationships, reported some degree of conflict with their romantic partners due to the spread of COVID-19 and its related restrictions (24). Also, one recent study in China indicate that the particular dynamics of the COVID-19 pandemic and associated mitigation measures may cause an increase in relationship conflict and potentially lead to worse emotional well-being among partnered individuals (25).

Taken together, these results suggest that the COVID-19 lockdown affects both the mechanisms of parenting and family life and the interplay between parents' well-being on children's adjustment. However, so far, mainly cross-sectional studies have been carried out to conduct parent-perceived stress among the parental population during COVID-19 lockdown $(4,23)$. Additional longitudinal studies, as presented in the current study, offer the benefit of examining changes of parents-perceived stress and predictors of these changes at several time points.

The present study seeks to add to the literature on parent-perceived stress in a two-wave longitudinal survey. Specifically, the purpose of the present study was to examine the impact of the corona crisis on parent's psychological well-being by investigating the change in perceived parental stress and symptoms of anxiety and depression among parents at two time points: 1) during the strict government initiated physical distancing protocols following the onset of the COVID-19 pandemic (T1) and 2) after the distancing protocols were discontinued, three months after the protocols were effectuated (T2). Furthermore, we 
Running head: Mechanisms of Parental Stress

examined the levels of parental stress, anxiety, and depression relative to variables related to family dynamics, such as relationship quality and anger aimed at child(ren) at the two time points.

First, we hypothesized that there would be a significant decrease in parental stress among parents from $\mathrm{T} 1$ to $\mathrm{T} 2$. Levels of parent perceived stress across different demographic subgroups were further investigated.

Second, we hypothesized that higher levels of anxiety and depressive symptoms, lower relationship quality and anger aimed at child(ren), together with less reduction in anxiety and depressive symptoms and less reduction in anger aimed at child, would be associated with less reduction in parental stress from $\mathrm{T} 1$ to $\mathrm{T} 2$, above and beyond the influence of demographic variables (i.e., gender, age, and the number of children in the household). 


\section{Methods}

\section{Participants}

The data for the current study originates from a two-wave longitudinal study of parental stress among parents three months after the pandemic outbreak in Norway (T2), compared to levels of parental stress in the same sample in the midst of the strictest government-initiated social distancing protocols (T1). At time point 1 (T1), participants included 2,868 parents, 21 to 83 years old $\left(M_{\mathrm{age}}=39.8, S D=8.0\right)$. The majority of the sample was female $(79.4 \%, n=2278)$ and Norwegian-born $(91.7 \%, n=2631)$. In terms of family structure, $80.4 \%(n=2305)$ of the parents reported as married or in a civil union. Also, $83.9 \%$ of parents reported living with one child per parent or less, and $16.1 \%$ reported living with more than one child per parent in the household. Out of the participating parents at T1 ( $n=2278$ mothers; $n=587$ fathers), $81.7 \%$ of mothers and $68.5 \%$ of fathers reported to have a higher education (completed a university degree or currently studying). Also, $17.2 \%(n=391)$ of mothers and $10.4 \%(n=$ 61) of fathers at T1 reported a pre-existing psychiatric diagnosis, which reflects the lower end of the known rate of psychological disorders in the adult population of Norway, which is between $16.66 \%$ to $25.00 \%$ (26).

At time point $2(\mathrm{~T} 2), 52 \%(n=1489)$ of the parents who participated at T1 continued to take part in the study. The current sample ranged from 24 to 69 years of age $\left(M_{\text {age }}=40.7\right.$ $S D=7.3)$, and the majority of the sample was still female $(80.3 \%, n=1195)$ and Norwegianborn $(92.7 \%, n=1381)$. Also, $82.8 \%(n=1233)$ of the parents reported as married or in a civil union. Out of the participating parents at $\mathrm{T} 2,84.5 \%$ of parents reported to live with one child per parent or less, and $15.5 \%$ reported to live with more than one child per parent in the 
Running head: Mechanisms of Parental Stress

household. Furthermore, out of the sample ( $n=1195$ mothers; $n=293$ fathers $)$ at T2, $85.9 \%$ of mothers and $76.5 \%$ of fathers reported to have completed a university degree or currently studying at the university. Also, $16.0 \%(n=191)$ of mothers and $9.6 \%(n=28)$ of fathers reported a pre-existing psychiatric diagnosis. Table 1 provides characteristics of the parental sample at $\mathrm{T} 1$ and $\mathrm{T} 2$.

\section{Recruitment and Procedures}

The present study has a pre-post survey design, where participants were asked to fill out a set of validated questionnaires on two measurement occasions. As further described in the measures section, some questionnaires were given as a whole, whereas other questionnaires included theoretically driven selections of items from validated questionnaires through a consensus process involving clinical experts with the purpose of not overwhelming the participants with an extensively long survey.

The first time point of data collection (T1) lasted seven days and was undertaken between March 31 and April 7, which encompasses a timeframe in which all governmentinitiated psychical distancing protocols were held constant during the data collection week, as well as two weeks before data collection. No information was given from the government about possible changes in any epidemic protocols during the data-collection period, controlling for expectation effects. All the parents who participated at T1 $(N=2,868)$ were invited to participate at the second wave of data (T2), which was measured between June 22 and July 13. This initiation date for measurement at $\mathrm{T} 2$ is exactly two weeks after government officials informed about the upcoming removal the preponderance of physical distancing protocols, which had been in place since March 12 . On June 22, the majority of pandemic mitigations protocols were discontinued in Norway. From T1 to T2, the corresponding attrition rate for parent participants was $48.1 \%$. There was no monetary compensation for participating. 
Running head: Mechanisms of Parental Stress

To give the parental population an equal opportunity to participate in the study, the survey was primarily disseminated through a Facebook Business algorithm to any adult (i.e., 18 years or above) residing in Norway. This algorithm disseminates the survey to a random sample of the proportion of the adult population available on Facebook (i.e., $85 \%$ of the entire adult in Norway). Seventy percent of the overall participants included in this study are obtained through this random selection technique. To reach the residual $15 \%$ of adults that were not on this platform, the survey was disseminated systematically through national, regional and local platforms (i.e., newspapers, radio stations, and television) across the entire country. Only one of these platforms (i.e., national television) had 1.1 million viewers at the time of broadcast. Thus, with this wide dissemination technique, we estimate to have reached the adult population and provided its members with an equal probability of participating in the survey.

Inclusion criteria were all adults 18 years of age and above, currently living in Norway and thus experiencing identical government-initiated physical distancing protocols, and who had provided digital consent to take part in the study. Exclusion criteria were children and adolescents (individuals below 18) and adults not residing in Norway during the measurement period. The stopping rule for data collection was designed to ensure that the government initiated physical distancing protocols were held constant across all counties for two weeks before and the week during the data collection period, as well as controlling for expectation effects by stopping data collection instantly once information concerning modification of government-initiated protocols was given. Thus, the stopping rule involved ending collection immediately if government-initiated protocols were changed or if novel information about forthcoming modifications was given (27). As the survey was administered online to a random selection of adults, it was not possible for us to stratify beforehand variables such as 
Running head: Mechanisms of Parental Stress

gender, proportion of marital status and education level. Consequently, we conducted poststratifications.

Data reported in this study are part of a longitudinal research project; The Norwegian COVID-19, Mental Health and Adherence Project, investigating the psychological impact of the COVID-19 pandemic in the Norwegian population during the strict government initiated physical distancing protocols related to the COVID-19 pandemic (T1) and three months after the pandemic breakout (T2). The discrepancy between $\mathrm{T} 1$ and $\mathrm{T} 2$ involves the discontinuation and reduction of overall numbers of physical distancing protocols from $\mathrm{T} 1$ to $\mathrm{T} 2$, with some mitigation protocols being terminated and others being softened. The pre-registered protocol for the present study can be found at Clinicaltrials.gov (Identifier: NCT04442308). Ethical approval of the study was granted by The Regional Committee for Medical and Health Research Ethics (reference number 125510) and The Norwegian Center for Research Data (reference number 802810).

\section{Measures}

Demographic background. Age, gender, cultural background, civil status, parents' education levels, number of children in household, employment status, and whether the participants had a self-reported pre-existing psychiatric diagnosis were assessed.

Perceived parenting stress. Parents perceived parenting stress was measured using the 18item Danish Parental Stress Scale (DPSS; 28). The DPSS is divided into two subscales: nine items measuring parental stress (e.g., "I feel overwhelmed by the responsibility of being a parent.") and seven items aimed to measure the lack of parental satisfaction (e.g., "I enjoy spending time with my children."). Three items from the parental stress subscale were chosen by a panel of clinical experts, with the aim of avoiding topological overlap and overburdening of participants. The items included were 1) "I feel overwhelmed by the responsibility of being 
Running head: Mechanisms of Parental Stress

a parent," 2) "The major source of stress in my life is my child(ren)," and 3) "It is difficult to balance different responsibilities because of my child(ren)." Parents reported the extent to which they agreed with the three items during the past month on a five-point scale ranging from 1 (strongly disagree) to 5 (strongly agree). The DPSS has shown good convergent and divergent validity in prior samples (28). In the present dataset, the internal reliability of the PSS items was good, with a Cronbach's alpha of 0.81 at T2 and 0.78 at T1.

Depression. Parents' depressive symptoms were assessed with the nine-item Patient Health Questionnaire (PHQ-9; 29). This measure is routinely used to assess symptoms of depression in accordance with the diagnostic criteria for major depression disorder and consists of nine items where each is scored on a four-point Likert scale ( 0 ; not at all to 3 ; almost every day). Higher scores indicate more depressive symptoms, and scores from ten and above represent a cut-off point for having symptoms associated with a major depressive disorder of moderate degree. Research supports the validity of the questionnaire to measure depressive symptoms in the general population (30).

Anxiety. The Generalized Anxiety Disorder 7 (GAD-7; 31) was used to obtain symptoms of anxiety and worry, encompassing seven items scored on a four-point Likert scale, ranging from 0 (not at all) to 4 (almost every day). The scale has proven to be a valid and reliable measure of anxiety symptoms in the general population (e.g., 31) and ten was used as cut-off.

Relationship satisfaction. Relationship satisfaction was measured by asking respondents whether they "Were more content with their relationship since the pandemic outbreak" (In Norway: middle of March).

Angry at child. Angry at child was measured by asking respondents whether they during the last two weeks "Have been angrier and more frustrated then usual at my children". 


\section{Statistical analysis}

Repeated surveys like the present one typically have a lot of drop out and missing data.

Therefore, data was analyzed with mixed models with maximum likelihood estimation, which is the state-of-the-art approach for handling missing data (32). Especially if data are missing at random, which is likely in our survey, mixed models give more unbiased results than the other analytic methods (33).

In preliminary analysis for parental stress as the dependent variable, a model with a random intercept, heteroskedasticity was modeled with a diagonal covariance structure. Akaike's Information Criterion (AIC) was used to compare the fit of different models. Models that gave a reduction in AIC greater than 2 were considered better (34).

First, $\mathrm{H} 1$ about a decrease in parental stress was tested by using parental stress as a dependent variable in a model using time $(\mathrm{T} 1$ period $=0, \mathrm{~T} 2$ period $=1)$ as a predictor. Second, demographic group variables will be added as predictors. Only those demographic variables found to be significant will be kept in subsequent analyses. Third, the initial (T1) levels of angry at child, anxiety and depression will be added, together with the interactions of these constant covariates with time. Relationship quality during the whole pandemic period, measured at T2, is considered a constant and is thus included as a predictor. These interactions represent tests of $\mathrm{H} 2$ about the covariates predicting change in parental stress, called Model 1. Finally, the T2 angry at child, anxiety and depression as constant covariates will be added, together with the interactions of these constant covariates with time. These 
Running head: Mechanisms of Parental Stress

interactions represent tests of $\mathrm{H} 2$ about the change in the covariates from $\mathrm{T} 1$ to $\mathrm{T} 2$, predicting a change in parental stress from T1 to T2, called Model 2.

\section{Results}

The mean level of perceived parental stress among the 2,868 parents was $7.1(S D=3.2)$ at the first time point (T1), compared to $6.4(S D=3.0)$ at the second time point $(\mathrm{T} 2)(n=1,489)$. Table 2 shows the demographic information of the participants and the mean level of parental stress in each subgroup. A paired samples t-test revealed a significant difference between the means at T1 and T2 $(t=10.344, p<.001)$. Furthermore, an analysis of variance (ANOVA) found significant differences in the mean level of parental stress between age groups at $\mathrm{T} 2$, and two independent t-tests revealed significant effects of being unemployed and having a pre-existing psychiatric diagnosis on the mean level of parenting stress at T2. Three participants with age $65+$ were not included in the analysis.

With regard to the reported symptoms of depression (PHQ-9) among the participating parents, $23.0 \%$ had symptoms meeting the clinical cut-off for depression at $\mathrm{T} 1$, as compared to $16.8 \%$ at $\mathrm{T} 2$. Also, at $\mathrm{T} 1,23.3 \%$ had symptoms meeting the clinical cut-off for generalized anxiety disorder (GAD-7), as compared to $13.8 \%$ at $\mathrm{T} 2$. These findings suggest a significant decline in both depressive symptoms $(t=5.72, p<.001)$ and anxiety symptoms $(t=8.93, p$ $<.001)$ across the two time points. Table 4 provides information about the number of parents meeting the clinical cut-off for depression and anxiety at T1 and T2. 


\section{Multilevel Models}

As hypothesized, there was a significant decrease in parental stress among parents from time point 1 (T1), where the government-initiated physical distancing protocols were held constant, to time point 2 (T2), where the preponderance of distancing protocols discontinued $(B=-0.72, S E=0.07, t(1707)=-10.87, p<.001)$.

Demographic variables, using age, gender, and the number of children, were entered as predictors of parental stress. The interaction of number of children with time was not related to parental perceived stress, that is, number of children was not significantly related to the change of parental stress, $B=-0.10, S E=0.08, t(1691)=-1.28, p=.200$.

However, the interaction of age with time, indicated that higher age led to less decrease in parental stress, $B=0.03, S E=0.01, t(1693)=2.61, p<.01$. There was a trend for the interaction of gender with time, indicating less decrease for males, $B=0.36, S E=0.17$, $t(1693)=2.13, p=0.034$. Since only age had a significant interaction with time at our predefined significance level $(p<0.01)$, only age was used as a control variable in the subsequent analyses.

Table 5 shows the results of the T1 predictors. As shown in Model 1 and as hypothesized, the interaction of relationship quality and anger at child(ren) at T1 with time predicted parental stress; however, the level of anxiety and depression at T1 was not related to a change in parental stress. As shown in Model 2 and as hypothesized, depression and angry 
Running head: Mechanisms of Parental Stress

at child at $\mathrm{T} 2$ predicted less reduction of parental stress. Thus, reduction on these variables from $\mathrm{T} 1$ to $\mathrm{T} 2$ are associated with a reduction in perceived parental stress. However, this pattern was not found for anxiety.

\section{Discussion}

The present study investigated the levels of perceived stress and symptoms of depression and anxiety among parents in the midst of the strictest distancing protocols (T1), compared to after the protocols were discontinued (T2). As expected, the levels of parental stress significantly decreased from $\mathrm{T} 1$ to $\mathrm{T} 2$, indicating that the overall level stress among the parents declined as a function of the phaseout of the distancing protocols. The decrease of perceived parental stress at the two time points, was accompanied by a significant decrease in symptoms of both depression and anxiety among the participating parents.

Decrease in perceived parental stress, as well as anxiety and depression from T1 to T2, may appear as a function of perceived controllability among the parents, as T2 represents a time point where the controllability regarding the quarantine duration was clarified (i.e., schools and daycares had re-opened after the lockdown period). This finding is in line with earlier studies suggesting that perceived control in stressful life events are associated with psychological well-being $(21,35)$ and decrease in overall stress, anxiety, and depression $(36$, $37)$.

Repeal of government-initiated distancing protocols may include fuller information regarding the mitigation situation, which may contribute to reducing the perceived stress and anxiety that may be related to inadequate information and lack of transparency from public authorities. Inadequate information from health and government authorities may be a major 
Running head: Mechanisms of Parental Stress

stressor because it leads to confusion regarding the importance and rationale of the physical distancing protocols $(38,39)$. Coping with the stress may be easier when the uncertainty of the quarantine duration is clarified and the perceived controllability related to the quarantine is restored, nevertheless other circumstances regarding the pandemic are not yet illuminated. Importantly, not all of the participating parents in the present study reported to experience high levels of parental stress during the strictest distancing protocols, although the protocols were collectively experienced by the parents. This finding is in line with existing evidence, which suggest that not all are at risk of higher perceived stress although they were exposed to same stressful life events (40). Individuals appraise events differently and individual responses vary according the subjective perception of the stressors, as well as to different coping strategies $(40,41)$. According to Lazarus \& Folkman (40) this evaluation involves estimating the resources available and the most effective strategies to deal with the situation, which is a key element of this assessment is the extent to which the individual can maintain control over the outcome of the situation. Additionally, a range of protective factors may serve as a buffer for families experiencing stressful events (42).

Indeed, the present study, supports such notions, since relationship satisfaction was found to predict change in parental stress. This finding is in accordance with the myriad of literature, which highlights relationship quality- and satisfaction as a significant protective factor. Several studies indicate that relationship satisfaction and supportive family environments is strongly associated with individual psychological well-being and lower levels of stress, see (43) for a meta-analysis. Relationship satisfaction is also considered a vital source of emotional and psychological well-being when families are experiences social crisis, as the ongoing pandemic (19). Some studies also indicate that highly stressful life events, such as natural disasters, may increase relationship quality $(17,18)$. 
Running head: Mechanisms of Parental Stress

Interestingly, as indicated in Model 1, angry aimed at child(ren) at T1, was found to predict change in parental stress. This finding is in line with findings revealed in studies where stress stemming from parent-child interaction has been studied specifically with relation to anger expression. Increased parenting stress has repeatedly been identified as a risk factor for maladaptive parenting practices, see (44) for a review). A predominant finding in several studies is that level of parental stress has been reported to be associated with abusive parenting (e.g., 45) and anger expression aimed at children $(46,47)$. For example, Rodriguez \& Green (45) found that parenting stress and anger expression were correlated with child abuse potential. Interestingly, the two factors combined further predicted abuse scale scores, which indicate that both stress and anger expression was found to contributing significant to the levels of stress (45). Another study reported that anger expression and parental stress were found to be predictive of child abuse potential and physical aggression toward children (47).

However, studies which indicate a robust link between levels of parental stress and anger expression, are accompanied by studies which shows that child anger proneness and child emotion dysregulation predicts parenting stress (48). These findings are particular interesting, seen in light of the stressful life event the ongoing pandemic represent, where child anger proneness and child disruptive behavior problem may be a way children are reacting at the physical distancing protocols (49), that in turn may contribute to the patterns seen in recent studies covering the field, as well as the present study. The association between perceived stress and anger need not to be considered unidirectional, as expressions of anger may create stressful situations and vice versa. This mechanism may also be present in the finding shown in Model 2, which indicate that reduction in both angry at child(ren) and depression from $\mathrm{T} 1$ to $\mathrm{T} 2$ was associated with a reduction in perceived parental stress. This finding may underline the fact that parental stress is driven by several possible mechanisms, including parental depression (50). 


\section{Conclusions}

Overall, the findings underline some of the negative psychological impact of the physical distancing protocols on parent's health and well-being. As expected, the levels of parental stress, depression and anxiety in the parental sample significantly decreased as a function of the phaseout of the distancing protocols. These results support earlier findings that quarantine increases susceptibility to stress and may have harmful effects on mental health, as seen in the general population $(21,51,52,53)$ and in particular among parents $(4,23,22)$. Our findings are also consistent with recent published studies, which indicate that parents experienced cumulative stressors due to COVID-19 and that a majority of parents reported experiencing symptoms of anxiety and depression (4). Parents who are faced with competing demands of limiting social interactions and remaining at home with their children may be particularly vulnerable during this time psychological distress as an important side effect of mass-quarantine. With regards to the future research, multiple timepoints are needed to unlock the patterns between level of parental stress and factors such as relationship quality and anger expression. Uncovering the nature of how these constructs are associated in relation to social crisis in particular, can contribute to design relevant interventions to reduce parental stress.

\section{Strengths and Limitations}

A major strength of this paper includes capturing the detrimental effects associated with government-initiated distancing protocols applied globally, making its findings generalizable across similar cultures employing similar distancing protocols. Another strength of the present study includes the large sample of parents experiencing identical interventions across the two measurement periods. Although, the sample was largely comprised of well-educated female 
Running head: Mechanisms of Parental Stress

participants and a small sample size of men, which may indicate a bias in the sample, the sensitivity analysis has shown robust results (27). A limitation of this study is that it is based on self-reported measures. In addition, since the present study includes a cross-sectional design, the ability to draw conclusions concerning directional and causal effects are impaired.

\section{References}

1. Prime H, Wade M, Browne DT. Risk and resilience in family well-being during the COVID-19 pandemic. Am Psychol. (2020) 75(5):631-43. doi: 10.1037/amp0000660

2. Vinkers CH, van Amelsvoort T, Bisson JI, Branchi I, Cryan JF. Domschke K, et al. Stress resilience during the coronavirus pandemic. Eur Neuropsychopharmacol. (2020) 35:12-16. doi: 10.1016/j.euroneuro.2020.05.003

3. Horesh D, Brown AD. Traumatic stress in the age of COVID-19: A call to close critical gaps and adapt to new realities. Psychol Trauma. (2020) 12(4):331-35. doi: $10.1037 / \operatorname{tra} 0000592$

4. Brown SM, Doom JR, Lechuga-Peña S, Watamura SE, Koppels T. Stress and parenting during the global COVID-19 pandemic. Child Abuse Negl. (2020) 104699. doi: 10.1016/j.chiabu.2020.104699.

5. Crasta D, Daks JS, Rogge RD. Modeling suicide risk among parents during the COVID-19 pandemic: Psychological inflexibility exacerbates the impact of COVID19 stressors on interpersonal risk factors for suicide. J Contextual Behav Sci. (2020) 18:117-27. doi: 10.1016/j.jcbs.2020.09.003 
Running head: Mechanisms of Parental Stress

6. Jiao WY, Wang LN, Liu J, Fang SF, Jiao FY, Pettoello-Mantovani M, et al. Behavioral and emotional disorders in children during the COVID-19 epidemic. $J$ Pediatr. (2020) 221:264-66.e1. doi: 10.1016/j.jpeds.2020.03.013

7. Gassman-Pines A, Ananat OE, Fitz-Henley J. COVID-19 and parent-child psychological well-being. Pediatr. (2020) 146(4):e2020007294. doi: 10.1542/peds.2020-007294

8. Griffith AK. Parental burnout and child maltreatment during the COVID-19 pandemic. J Fam Violence. (2020) 23:1-7. doi: 10.1007/s10896-020-00172-2

9. Patrick SW, Henkhaus LE, Zickafoose JS, Lovell K, Halvorson A, Loch S, et al. Wellbeing of parents and children during the COVID-19 pandemic: A national survey. Pediatr. (2020) 146(4):e2020016824. doi: 10.1542/peds.2020-016824

10. Peltz JS, Daks JS, Rogge RD. Mediators of the association between COVID-19related stressors and parents' psychological flexibility and inflexibility: The roles of perceived sleep quality and energy. J Contextual Behav Sci. (2020) 17:168-76. doi: 10.1016/j.jcbs.2020.07.001

11. Campbell AM. An increasing risk of family violence during the Covid-19 pandemic: Strengthening community collaborations to save lives. FSI Reports. (2020) 2:100089. doi: 10.1016/j.fsir.2020.100089

12. Usher K, Bhullar N, Durkin J, Gyamfi N, Jackson D. Family violence and COVID-19: Increased vulnerability and reduced options for support. Int J Ment Health Nurs. (2020) 29(4):549-52. doi: 10.1111/inm.12735

13. Deater-Deckard K D. Parenting stress. (2004). New Haven, CT: Yale University Press.

14. Abidin RR. The determinants of parenting behavior. J Clin Child Psychol. (1992) 21(4):407-12. doi: 10.1207/s15374424jccp2104_12 
Running head: Mechanisms of Parental Stress

15. Bradbury-Jones C, Isham L. The pandemic paradox: The consequences of COVID-19 on domestic violence. J Clin Nurs. (2020) 29(13-14):2047-49. doi:

10.1111/jocn. 15296

16. Beckerman M, van Berkel SR, Mesman J, Alink LRA. The role of negative parental attributions in the associations between daily stressors, maltreatment history, and harsh and abusive discipline. Child Abuse Negl. (2017) 64:109-16. doi:

10.1016/j.chiabu.2016.12.015

17. Fredman SJ, Monson CM, Schumm JA, Adair KC, Taft CT, Resick PA. Associations among disaster exposure, intimate relationship adjustment, and PTSD symptoms: Can disaster exposure enhance a relationship? J Trauma Stress. (2010) 238(4):446-51. doi: $10.1002 /$ jts. 20555

18. Marshall EM, Kuijer RG. Weathering the storm? The impact of trauma on romantic relationships. Curr Opin Psychol. (2017). 13:54-59 doi: 10.1016/j.copsyc.2016.04.013

19. Pietromonaco PR, Overall NC. Applying relationship science to evaluate how the COVID-19 pandemic may impact couples' relationships. Am Psychol. (2020) Advance online publication. doi: 10.1037/amp0000714

20. Chan KL, Zhang Y. Female victimization and intimate partner violence after the May 12, 2008, Sichuan earthquake. Violence Vict. (2011) 26(3). doi: 10.1891/08866708.26.3.364.

21. Brooks SK, Webster RK, Smith LE, Woodland L, Wessely S, Greenberg, N, et al. The psychological impact of quarantine and how to reduce it: Rapid review of the evidence. Lancet. (2020) 395(10227):912-20. doi: 10.1016/S0140-6736(20)30460-8

22. Sprang G, Silman M. Posttraumatic stress disorder in parents and youth after healthrelated disasters. Disaster Med Public Health Prep. (2013) 7(1):105-10. doi: $10.1017 / \mathrm{dmp} .2013 .22$ 
Running head: Mechanisms of Parental Stress

23. Spinelli M, Lionetti F, Pastore M, Fasolo M. Parents stress and children's psychological problems in families facing the COVID-19 outbreak in Italy. Front Psychol. (2020) 11:1713 doi: 10.3389/fpsyg.2020.01713

24. Luetke M, Hensel D, Herbenick D, Rosenberg M. Romantic relationship conflict due to the COVID-19 pandemic and changes in intimate and sexual behaviors in a nationally representative sample of American adults. J Sex Marital Thera. (2020) 46(8):747-62. doi: 10.1080/0092623X.2020.1810185

25. Yang H, Ma J. How an epidemic outbreak impacts happiness: Factors that worsen (vs. protect) emotional well-being during the coronavirus pandemic. Psychiatry Res. (2020) 289: Article 113045. doi: 10.1016/j.psychres.2020.113045

26. Norwegian Institute of Public Health. Mental Illness Among Adults in Norway. Public Health Reports - Health Status in Norway (2016). Available online at:

27. Ebrahimi OV, Hoffart A, Johnson SU. The mental health impact of nonpharmacological interventions aimed at impeding viral transmission during the COVID-19 pandemic in a general adult population and the factors associated with adherence to these mitigation strategies. (2020, May 9). doi: 10.31234/osf.io/kjzsp

28. Pontoppidan M, Nielsen T, Kristensen IH. Psychometric properties of the Danish Parental Stress Scale: Rasch analysis in a sample of mothers with infants. PLoS One. (2018) 13(11):e0205662. doi: 10.1371/journal.pone.0205662

29. Kroenke K, Spitzer RL, Williams JBW. The PHQ-9: Validity of a brief depression severity measure. J Gen Intern Med. (2001) 16(9):606-13. doi: 10.1046/j.15251497.2001.016009606.x

30. Martin A, Rief W, Klaiberg A, Braehler E. Validity of the Brief Patient Health Questionnaire Mood Scale (PHQ-9) in the general population. Gen Hosp Psychiatry. (2006) 28(1):71-77. doi: 10.1016/j.genhosppsych.2005.07.003 
Running head: Mechanisms of Parental Stress

31. Spitzer RL, Kroenke K, Williams JBW, Löwe B. A brief measure for assessing generalized anxiety disorder: The GAD-7. Arch Intern Med. (2006) 166(10):1092-97. doi: 10.1001/archinte.166.10.1092

32. Schafer JL, Graham JW. Missing data: Our view of the state of the art. Psychol Methods. (2002) 7(2):147-77. doi: 10.1037/1082-989X.7.2.147

33. O’Connell NS, Dai L, Jiang Y, Speiser JL, Ward R, Wei W, et al. Methods for analysis of pre-post data in clinical research: A comparison of five common methods. J Biom Biostat. (2017) 8(1):334. doi: 10.4172/2155-6180.1000334

34. Burnham KP, Anderson DR. Multimodel inference: Understanding AIC and BIC in model selection. Sociol Methods Res. (2004) 33(2):261-304. doi: $10.1177 / 0049124104268644$

35. Dijkstra MTM, Homan AC. Engaging in rather than disengaging from stress: Effective coping and perceived control. Front Psychol. (2016) 7:1415. doi: 10.3389/fpsyg.2016.01415

36. Ballash NG, Pemble MK, Usui WM, Buckle, AF, Woodruff-Borden J. Family functioning, perceived control, and anxiety: A mediational model. J Anxiety Disord. (2006) 20(4):486-97. doi: 10.1016/j.janxdis.2005.05.002

37. Compas BE, Banez GA, Malcarne V, Worsham N. Perceived control and coping with stress: A developmental perspective. J Soc Issues. (1991) 47(4):23-43. doi: 10.1111/j.1540-4560.1991.tb01832.x

38. Cava MA, Fay KE, Beanlands HJ, McCay EA, Wignall R. The experience of quarantine for individuals affected by SARS in Toronto. Public Health Nurs. (2005) 22(5):398-406. doi: 10.1111/j.0737-1209.2005.220504.x 
Running head: Mechanisms of Parental Stress

39. Serafini G, Parmigianin B, Amerio A, Aguglia A, Sher L, Amore L. The psychological impact of COVID-19 on the mental health in the general population. QJM. (2020) 113(8):531-37. doi: 10.1093/qjmed/hcaa201

40. Lazarus RS, Folkman S. Stress, appraisal, and coping. Springer (1984)

41. Folkman S. Stress, coping and hope. Psycho-Oncology. (2010) 19:901-08. doi: 10.1002/pon.1836

42. Hjemdal O, Friborg O, Stiles TC, Rosenvinge JH, Martinussen M. Resilience predicting psychiatric symptoms: A prospective study of protective factors and their role in adjustment to stressful life events. Clin Psychol Psychother. (2006) 13(3):194 201. doi: $10.1002 /$ cpp.488

43. Falconier MK, Jackson JB, Hilpert P, Bodenmann G. Dyadic coping and relationship satisfaction: A meta-analysis. Clin Psychol Rev. (2015) 42:28-46. doi: 10.1016/j.cpr.2015.07.002.

44. Kazdin AE, Whitley MK. Treatment of parental stress to enhance therapeutic change among children referred for aggressive and antisocial behavior. J Consult Clin Psychol. (2003) 71(3):504-15. doi: 10.1037/0022-006X.71.3.504

45. Rodriguez CM, Green AJ. Parenting stress and anger expression as predictors of child abuse potential. Child Abuse Negl. (1997) 21(4):367-77. doi: 10.1016/S01452134(96)00177-9

46. Lam D. Parenting stress and anger: The Hong Kong experience. Child Fam Soc Work. (2001) 4(4). doi: 10.1046/j.1365-2206.1999.00133.x

47. Rodriguez CM, Richardson MJ. Stress and anger as contextual factors and preexisting cognitive schemas: Predicting parental child maltreatment risk. Child Maltreat. (2007) 12(4):325-37. doi: 10.1177/1077559507305993 
Running head: Mechanisms of Parental Stress

48. Williford AP, Calkins SD, Keane SP. Predicting change in parenting stress across early childhood: Child and maternal factors. J Abnorm Child Psychol. (2007) 35:25163. doi: 10.1007/s10802-006-9082-3

49. Mondragon NI, Sancho NB, Santamaria MD, Munitis AE. Struggling to breathe: a qualitative study of children's wellbeing during lockdown in Spain. Psychol Health, (2020). 7, 1-16. doi: 10.1080/08870446.2020.1804570.

50. Webster-Stratton C, Hammond M. Maternal depression and its relationship to life stress, perceptions of child behavior problems, parenting behaviors and child conduct problems. J Abnormal Child Psychol (1988). 16(3):299-315. doi:

10.1007/BF00913802

51. Hawryluck L, Gold WL, Robinson S, Pogorski S, Galea S, Styra R. SARS control and psychological effects of quarantine, Toronto, Canada. Emerg Infect Dis. (2004) 10(7):1206-12. doi:10.3201/eid1007.030703

52. Hawkley LC, Cacioppo JT. Loneliness matters: A theoretical and empirical review of consequences and mechanisms. Ann Behav Med. (2010) 40(2):218-27. doi: $10.1007 / \mathrm{s} 12160-010-9210-8$

53. Hoffart A, Urnes Johnson S, Ebrahimi Ov. Loneliness and social distancing during the COVID-19 pandemic: Risk factors and associations with psychopathology. Front.Psychiatry. (2020). doi:10.3389/fpsyt.2020.589127 
Running head: Mechanisms of Parental Stress

Table 1

Demographic Characteristics of the Study Sample

\begin{tabular}{|c|c|c|}
\hline & Parents (T1) & Parents (T2) \\
\hline Characteristic & $(n=2,868)$ & $(n=1,489)$ \\
\hline Age & $39.8(S D=8.0)$ & $40.7(S D=7.3)$ \\
\hline Number of child(ren) in household & $1.8(S D=0.8)$ & $1.8(S D=0.7)$ \\
\hline \multicolumn{3}{|l|}{ Gender } \\
\hline Female & $2278(79.4 \%)$ & $1195(80.3 \%)$ \\
\hline Male & $587(20.5 \%)$ & $293(19.7 \%)$ \\
\hline \multicolumn{3}{|l|}{ Cultural background } \\
\hline Norwegian & $2713(94.6 \%)$ & $1410(94.7 \%)$ \\
\hline 1st generation immigrant & $127(4.4 \%)$ & $66(4.4 \%)$ \\
\hline 2nd generation immigrant & $28(1.0 \%)$ & $13(0.9 \%)$ \\
\hline \multicolumn{3}{|l|}{ Civil Status } \\
\hline Single parent & $422(14.7 \%)$ & $206(13.8 \%)$ \\
\hline In a relationship & $141(4.9 \%)$ & $56(3.8 \%)$ \\
\hline Married/civil union & $2305(80.4 \%)$ & $1227(82.4 \%)$ \\
\hline \multicolumn{3}{|l|}{ Parent work status } \\
\hline Mother works (yes) & $1735(76.2 \%)$ & $930(77.8 \%)$ \\
\hline Father works (yes) & $503(85.7 \%)$ & $249(85.0 \%)$ \\
\hline
\end{tabular}


Running head: Mechanisms of Parental Stress

Table 2

Demographic Information of the Participants at T2 and Mean Level of Parental Stress in each subgroup

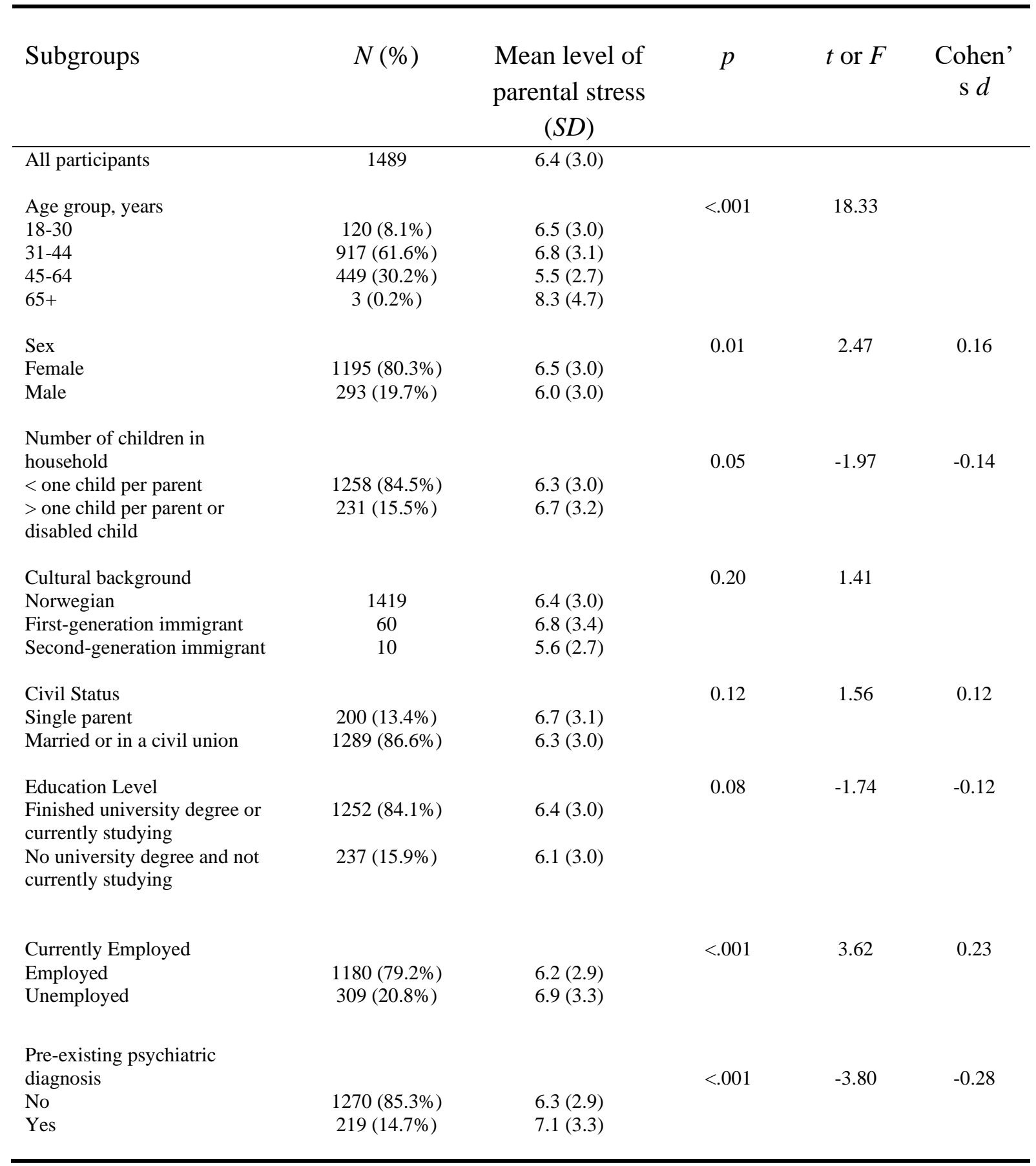


Running head: Mechanisms of Parental Stress

Table 3

Demographic Information of the Participants at T1 and Mean Level of Parental Stress in each subgroup

\begin{tabular}{|c|c|c|c|c|c|}
\hline Subgroups & $N(\%)$ & $\begin{array}{c}\text { Mean level of } \\
\text { parental stress } \\
(S D)\end{array}$ & $p$ & $t$ or $F$ & $\begin{array}{c}\text { Cohen' } \\
\text { s } d\end{array}$ \\
\hline All participants & & $7.1(3.1)$ & & & \\
\hline $\begin{array}{l}\text { Age group, years } \\
18-30 \\
31-44 \\
45-64 \\
65+\end{array}$ & $\begin{array}{c}359(12.5 \%) \\
1728(60.3 \%) \\
768(26.8 \%) \\
13(0.45 \%)\end{array}$ & $\begin{array}{l}7.3(3.1) \\
7.6(3.2) \\
5.9(2.8) \\
5.6(2.6)\end{array}$ & $<.001$ & 54.05 & \\
\hline $\begin{array}{l}\text { Sex } \\
\text { Female } \\
\text { Male }\end{array}$ & $\begin{array}{c}2278(79.5 \%) \\
587(20.5 \%)\end{array}$ & $\begin{array}{l}7.3(3.2) \\
6.4(2.9)\end{array}$ & $<.001$ & 5.91 & 0.27 \\
\hline $\begin{array}{l}\text { Number of children in } \\
\text { household } \\
<\text { one child per parent } \\
\text { > one child per parent or } \\
\text { disabled child }\end{array}$ & $\begin{array}{c}2407(83.9 \%) \\
461(16.1 \%)\end{array}$ & $\begin{array}{l}7.0(3.1) \\
7.6(3.2)\end{array}$ & $<.001$ & -4.00 & -0.20 \\
\hline $\begin{array}{l}\text { Cultural background } \\
\text { Norwegian } \\
\text { First-generation immigrant } \\
\text { Second-generation immigrant }\end{array}$ & $\begin{array}{c}2713 \\
127 \\
28\end{array}$ & $\begin{array}{l}7.1(3.2) \\
7.0(3.0) \\
6.6(2.8)\end{array}$ & 0.65 & 0.43 & \\
\hline $\begin{array}{l}\text { Civil Status } \\
\text { Single parent } \\
\text { Married or in a civil union }\end{array}$ & $\begin{array}{c}563(19.6 \%) \\
2305(80.4 \%)\end{array}$ & $\begin{array}{l}7.0(3.1) \\
7.1(3.1)\end{array}$ & 0.48 & -0.7 & -0.03 \\
\hline $\begin{array}{l}\text { Education Level } \\
\text { Finished university degree or } \\
\text { currently studying } \\
\text { No university degree and not } \\
\text { currently studying }\end{array}$ & $\begin{array}{l}2267(79.0 \%) \\
601(21.0 \%)\end{array}$ & $\begin{array}{l}7.2(3.2) \\
6.7(3.0)\end{array}$ & $<.001$ & -3.47 & -0.16 \\
\hline $\begin{array}{l}\text { Currently Employed } \\
\text { Employed } \\
\text { Unemployed }\end{array}$ & $\begin{array}{c}2241(78.1 \%) \\
627(21.9 \%)\end{array}$ & $\begin{array}{l}7.0(3.1) \\
7.3(3.3)\end{array}$ & 0.09 & 1.70 & 0.08 \\
\hline $\begin{array}{l}\text { Pre-existing psychiatric } \\
\text { diagnosis } \\
\text { No } \\
\text { Yes }\end{array}$ & $\begin{array}{c}2416(84.2 \%) \\
452(15.8 \%)\end{array}$ & $\begin{array}{l}7.0(3.1) \\
7.8(3.4)\end{array}$ & $<.001$ & -5.41 & -0.28 \\
\hline
\end{tabular}


Running head: Mechanisms of Parental Stress

Table 4

Participants in Different Subgroups Meeting the Diagnostic Cut-off Score for Depression and Anxiety Across Time Points

\begin{tabular}{|c|c|c|}
\hline \multirow{2}{*}{$\begin{array}{l}\text { Subgroups } \\
\text { Symptoms of depression } \\
\text { (PHQ-9) }\end{array}$} & \multicolumn{2}{|c|}{$\begin{array}{l}\text { No }(\%) \text { of participants meeting the } \\
\text { diagnostic cut-off at } \mathrm{T} 1 \text { and } \mathrm{T} 2\end{array}$} \\
\hline & T1 & T2 \\
\hline All participants & $659(23.0)$ & $250(16.8)$ \\
\hline $\begin{array}{l}\text { Sex } \\
\text { Female } \\
\text { Male }\end{array}$ & $\begin{array}{c}577(25.3) \\
81(13.8)\end{array}$ & $\begin{array}{c}213(17.8) \\
37(12.6)\end{array}$ \\
\hline $\begin{array}{l}\text { Age group, years } \\
18-30 \\
31-44 \\
45-64 \\
65+\end{array}$ & $\begin{array}{c}123(34.3) \\
420(24.3) \\
115(15.0) \\
1(7.7)\end{array}$ & $\begin{array}{c}33(27.5) \\
168(18.3) \\
49(10.9) \\
0\end{array}$ \\
\hline $\begin{array}{l}\text { Civil status } \\
\text { Single parent } \\
\text { Married or in a civil union }\end{array}$ & $\begin{array}{l}150(35.5) \\
509(20.8)\end{array}$ & $\begin{array}{c}53(25.7) \\
197(15.4)\end{array}$ \\
\hline $\begin{array}{l}\text { Number of children in } \\
\text { household } \\
<\text { one child per parent } \\
>\text { one child per parent or } \\
\text { disabled child }\end{array}$ & $\begin{array}{l}566(23.5) \\
93(20.2)\end{array}$ & $\begin{array}{l}212(16.8) \\
38(16.5)\end{array}$ \\
\hline $\begin{array}{l}\text { Symptoms of anxiety } \\
\text { (GAD-7) }\end{array}$ & & \\
\hline All participants & $667(23.3)$ & $205(13.8)$ \\
\hline $\begin{array}{l}\text { Sex } \\
\text { Female } \\
\text { Male }\end{array}$ & $\begin{array}{c}585(25.7) \\
82(14.0)\end{array}$ & $\begin{array}{c}171(14.3) \\
34(11.6)\end{array}$ \\
\hline $\begin{array}{l}\text { Age group, years } \\
18-30 \\
31-44 \\
45-64 \\
65+\end{array}$ & $\begin{array}{c}122(34.0) \\
434(25.1) \\
111(14.4) \\
0(0.0)\end{array}$ & $\begin{array}{c}22(18.3) \\
146(15.9) \\
37(8.2) \\
0(0.0)\end{array}$ \\
\hline
\end{tabular}


Running head: Mechanisms of Parental Stress

Single parent

Married or in a civil union

Number of children in

household

$<$ one child per parent

$>$ one child per parent or

disabled child
$135(32.0)$

$42(20.4)$

$532(21.8)$

$163(12.7)$

$571(23.7)$

$96(20.8)$

$171(13.6)$

$34(14.8)$ 
Running head: Mechanisms of Parental Stress

Table 5

Predictors of Parental Stress

\begin{tabular}{|c|c|c|c|c|c|c|}
\hline Predictor & Estimate & SE & $\mathrm{t}$ & Estimate & SE & $\mathrm{t}$ \\
\hline & \multicolumn{3}{|c|}{$\underline{\text { Model } 1}$} & \multicolumn{3}{|c|}{ Model 2} \\
\hline Intercept & 4.45 & 0.87 & $5.12 *$ & 5.57 & 0.91 & $6.14 *$ \\
\hline Time & 1.03 & 0.54 & $1.92 *$ & -0.77 & 0.53 & -1.47 \\
\hline Age & -0.06 & 0.02 & $-3.69 *$ & -0.07 & 0.02 & $-3.94 *$ \\
\hline Relationship & 0.13 & 0.11 & 1.12 & 0.01 & 0.12 & 0.07 \\
\hline Depression $^{1}$ & 0.15 & 0.04 & $3.96^{*}$ & 0.21 & 0.04 & $4.99 *$ \\
\hline Anxiety $^{1}$ & -0.06 & 004 & -1.44 & -0.04 & 0.05 & -0.87 \\
\hline Angry-child ${ }^{1}$ & 1.89 & 0.10 & $19.55^{*}$ & 2.05 & 0.11 & $19.28 *$ \\
\hline Time $\mathrm{X}$ age & 0.01 & 0.01 & 1.04 & 0.02 & 0.01 & 2.02 \\
\hline Time $X$ & -0.24 & 0.07 & $-3.37 *$ & -0.06 & 0.07 & -0.93 \\
\hline \multicolumn{7}{|l|}{ Relationship } \\
\hline Time $X$ & -0.06 & 0.02 & -2.39 & -0.12 & 0.03 & $-4.59 *$ \\
\hline \multicolumn{7}{|l|}{ Depression $^{1}$} \\
\hline Time $\mathrm{X}$ & 0.07 & 0.03 & 2.41 & 0.03 & 0.03 & 1.20 \\
\hline \multicolumn{7}{|l|}{ Anxiety $^{1}$} \\
\hline Time $X$ & -0.60 & 0.06 & $-10.10^{*}$ & -0.87 & 0.06 & $-14.29 *$ \\
\hline \multicolumn{7}{|l|}{ Angry-child ${ }^{1}$} \\
\hline Depression $^{2}$ & & & & -0.10 & 0.05 & -2.15 \\
\hline Anxiety $^{2}$ & & & & -0.01 & 0.06 & -0.07 \\
\hline Angry-child ${ }^{2}$ & & & & -0.43 & 0.13 & $-3.38^{*}$ \\
\hline Time $X$ & & & & 0.08 & 0.03 & $2.75^{*}$ \\
\hline \multicolumn{7}{|l|}{ Depression $^{2}$} \\
\hline Time $\mathrm{X}$ & & & & 0.03 & 0.03 & 0.59 \\
\hline \multicolumn{7}{|l|}{ Anxiety $^{2}$} \\
\hline Time $X$ & & & & 0.81 & +.08 & $10.76^{*}$ \\
\hline Angry-child ${ }^{2}$ & & & & & & \\
\hline
\end{tabular}

